Research Article

\title{
Screening for organic acidurias and aminoacidopathies in high-risk Brazilian patients: Eleven-year experience of a reference center
}

Moacir Wajner ${ }^{1,2}$ iD, Angela Sitta ${ }^{1}$, Aline Kayser ${ }^{1}$, Marion Deon ${ }^{1}$, Ana C. Groehs ${ }^{1}$, Daniella M. Coelho ${ }^{1}$ and Carmen R. Vargas ${ }^{1,3}$

${ }^{1}$ Serviço de Genética Médica, Hospital de Clínicas de Porto Alegre, Porto Alegre, RS, Brazil.

${ }^{2}$ Departmento de Bioquímica, Universidade Federal do Rio Grande do Sul, Porto Alegre, RS, Brazil.

${ }^{3}$ Programas de Pós-Graduação em Ciências Biológicas, Bioquímica e em Ciências Farmacêuticas, Universidade Federal do Rio Grande do Sul, Porto Alegre, RS, Brazil.

\begin{abstract}
Organic acidurias and aminoacidopathies are groups of frequent inborn errors of metabolism (IEMs), which are caused by mutations in specific genes that lead to loss of protein/enzyme or transport function with important deleterious effects to cell metabolism. Since a considerable number of such disorders are potentially treatable when diagnosed at an early stage of life, diagnosis is crucial for the patients. In the present report, we describe symptomatic individuals referred to our service that were diagnosed with these disorders from 2006 to 2016 . We used blood and urine samples from 21,800 patients suspected of aminoacidopathies or organic acidemias that were processed by the analytical techniques reverse phase high-performance liquid chromatography for amino acid quantification and gas chromatography coupled to mass spectrometry for organic acid detection. Analysis of dried blood spots by liquid chromatography-tandem mass spectrometry was used in some cases. We detected 258 cases of organic acidurias, and 117 patients with aminoacidopathies were diagnosed. Once diagnosis was performed, patients were promptly submitted to the available treatments with clear reduction of mortality and morbidity. The obtained data may help pediatricians and metabolic geneticists to become aware of these diseases and possibly expand newborn screening programs in the future.
\end{abstract}

Keywords: Organic acidurias, aminoacidopathies, inborn errors of metabolism, selective screening.

Received: April 7, 2018; Accepted: November 15, 2018.

\section{Introduction}

Inborn errors of metabolism (IEM) are phenotypically and genetically heterogeneous disorders caused by defects in specific proteins, mostly enzymes, which compromise cellular metabolism. Although any given IEM is rare when taken individually, they are collectively numerous, causing significant morbidity and mortality (Mak et al., 2013; Scaturro et al., 2013). Prevalence of these disorders is probably underestimated, except were populationbased neonate screening is conducted. More than 600 different IEMs have been already biochemically and molecularly identified. Disease state usually arises due to accumulation of toxic intermediate metabolites proximal to the metabolic block, lack of essential products, or accumulation of by-products (Scriver et al., 2001). Clinical manifestations usually occur in early infancy and childhood, with a heterogeneous clinical spectrum.

Send correspondence to Moacir Wajner. Serviço de Genética Médica, Hospital de Clínicas de Porto Alegre, Rua Ramiro Barcelos 2350, 90.035-003, Porto Alegre, RS, Brazil. E-mail: mwajner@hcpa.edu.br.
Some IEMs, including amino acid, organic acid, and fatty acid oxidation defects, can be treated with promising outcomes. Affected babies are usually normal at birth, but symptoms may develop within hours or weeks pending on the severity of the disease. The symptoms are often nonspecific, like poor feeding, seizures, and lethargy/coma that may progress to life-threatening complications if left untreated. Due to the serious clinical consequences for affected neonates, an early diagnosis allows pre-symptomatic treatment and is crucial in preventing irreversible mental retardation ranging from mild to severe, neurological damage, physical disability, and even death (Gramer et al., 2014). Diagnosis of these disorders can be performed also for some prevalent and treatable IEM in the asymptomatic babies through newborn mass screening (NBS) that was established in the last three decades, or for most of these disorders through selective screening in symptomatic patients (Leonard, 2006; Marsdeen et al., 2006).

Selective screening carried out in patients with clinical signs, alterations of routine laboratory tests, or family history indicating a metabolic disorder is an important di- 
agnostic tool for the diagnosis of IEM in general. These tests usually measure small molecules in intermediary metabolism disorders whose concentrations are elevated in biological fluids due to a block at some point of the metabolic pathway. Other tests detect large complex molecules accumulating in lysosomal disorders, which have been incorporated more recently in battery of tests for selective screening (Bravo et al., 2017). Improved diagnostic facilities for the detection of these disorders have increased the number of recognized IEM, as well as the discovery of new disorders. Early diagnosis is important not only for treatment but also for genetic counseling and prenatal diagnosis in future gestations. Late diagnosis and treatment of these metabolic disorders can cause permanent damage in essential tissues like the central nervous system (García-Gazorla et al., 2009). The techniques used most commonly for the detection of small metabolites are gas chromatography-mass spectrometry (GC-MS) for organic acids determination, reverse phase high performance liquid chromatography (HPLC) and ion exchange chromatography-postcolumn derivatization for amino acids measurement (Joseph and Marsden, 1986), and liquid chromatography-tandem mass spectrometry (LC-MS/MS) for amino acid and acylcarnitine analysis (Rashed et al., 1997). Basic metabolic tests comprise the assessment of urinary organic acids in urine, amino acids in plasma, urine and dried blood spots, as well as free carnitine and acylcarnitines in dried blood spots. The diagnosis is achieved by the identification of abnormal urinary organic acid profiling with elevated excretion of defined patterns of organic acids (organic acidurias), amino acids (aminocidopathies), and acylcarnitines (fatty acid oxidation defects) that are considered biomarkers for these classes of metabolic diseases.

Organic acidurias and aminoacidopathies are relatively frequent inherited disorders of intermediary metabolism resulting from deficiency of an enzyme or a transport protein involved in the catabolism of amino acids, carbohydrates, or lipids, leading to abnormal changes (usually increase) of physiological levels of organic acids and amino acids, respectively in the body and biological fluids (Van Vliet et al., 2014). They are considered the most prevalent metabolic diseases in severely ill children and the most prevalent groups of IEM (Chalmers et al., 1980). Many patients affected by these "intoxication" disorders have severe life-threatening symptoms with high morbidity and mortality. They typically suffer from either an acute lifethreatening illness in early infancy or unexplained developmental delay with recurrent episodes of metabolic decompensation in later childhood. A wide variety of symptoms and laboratory findings occur, such as lethargy, coma, hypotonia, seizures, ataxia, vomiting, failure to thrive, developmental delay, liver disease, neutropenia, thrombocytopenia, hypoglycemia, hyperammonemia, etc. The possibility of providing an adequate treatment apparently justifies the detection of these diseases in patients without established diagnosis.

The true incidence of aminoacidopathies and organic acidurias in high-risk Brazilian patients is still poorly established. In this study, we present the prevalence of these diseases in patients suspected of IEM during the past eleven years (2006-2016) that were referred to our service, which is a regional and national reference center for the diagnosis of these diseases. Our objective was to find out the most prevalent organic acid and amino acid disorders in a highrisk Brazilian population and to promote the awareness of these disorders within the medical community as important and prevalent groups of genetic diseases.

\section{Subjects and Methods}

\section{Patients}

The methodology for the diagnosis of organic acidurias and aminoacidopathies was set up in 1994 at the Medical Genetics Service of the Hospital de Clínicas de Porto Alegre (HCPA), in the city of Porto Alegre, Southern Brazil. We aimed to provide an adequate treatment for a considerable number of these disorders, justifying therefore their detection in patients without established diagnosis. Plasma $(1 \mathrm{~mL})$ and fresh random urine specimens (2-10 $\mathrm{mL}$ ) without preservative were collected and stored frozen within $2-3 \mathrm{~h}$ under $-30^{\circ} \mathrm{C}$. Sometimes cerebrospinal fluid (CSF) was collected and frozen under the same conditions or dried whole blood spots in filter paper we used for amino acid detection. This study was approved by the Ethical Committee of Hospital de Clínicas de Porto Alegre. Patients who participated in this study or their parents and/or guardians signed informed consent forms for collection of the biological samples.

Samples were collected from symptomatic 21,800 children (52\% boys and $48 \%$ girls) with suspected IEM or disorders of amino acid (plasma amino acid determination was requested in $48 \%$ of samples) or organic acid metabolism (organic acid detection was requested in $52 \%$ of urine samples), at the age varying from $24 \mathrm{~h}$ to 23 years from 17 Brazilian states (17/26 - 65.4\%) from public and private hospitals and outpatient clinics. Children less than 3 years old were $66 \%$ of the sample, and less than 12 months old were $40 \%$. Patients' clinical information was obtained from a questionnaire filled by health professionals. Neurological features $(81 \%)$, feeding refuse $(21 \%)$, hepatomegaly $(14 \%)$, dysmorphias (13\%), vomiting (11\%), and failure to thrive $(5 \%)$ were the most frequent clinical manifestations, whereas hypoglycemia $(15 \%)$, persistent or recurrent metabolic acidosis (13\%), and lactic acidemia (6\%) were the most common laboratory findings. Major neurological signs were unexplained psychomotor development/mental retardation $(39 \%)$, persistent seizures (32\%), abnormal muscular tone (22\%), macrocephaly $(3 \%)$, cerebral atrophy 
$(2 \%)$, lethargy/coma (1\%), and encephalopathic crises $(1 \%)$.

\section{Amino acid analysis}

The free amino acids in plasma, urine, or CSF were determined by reverse phase HPLC according to Joseph and Marsden (1986) with slight modifications (Wajner et al., 2000). Plasma and CSF were precipitated by methanol, while sodium tetraphenylborate was added to urine and the supernatants were stored at $-30{ }^{\circ} \mathrm{C}$ until analysis after centrifugation. A standard solution of amino acids was used for calibration (Sigma, Germany), prepared according to the manufacturer's protocol. The analysis was performed using a Shimadzu HPLC with a reverse phase column (ODS $25 \mathrm{~cm} \times 4.6 \mathrm{~mm} \times 5 \mu \mathrm{m}$ ) and fluorescent detection after pre-column derivatization with orthophthaldialdehyde plus mercaptoethanol. The flow rate was adjusted to 1.4 $\mathrm{mL} / \mathrm{min}$ in a gradient of the mobile phase (methanol and phosphate buffer: buffer A, $80 \%$ methanol; buffer B, $20 \%$ methanol). The duration of each analysis was $45 \mathrm{~min}$. Amino acids were quantitatively determined by relating their chromatographic peak area to those obtained from a known standard mixture and to that of internal standard peak area (homocysteic acid). The results were reported as $\mu \mathrm{mol} / \mathrm{L}$.

\section{Organic acid analysis}

Organic acids were determined in urine by GC/MS according to Sweetmann (2006), using hexadecane and heptadecanoic acid as internal standards. The organic acids were extracted twice with ethyl acetate and derived as bis(trimethyl-silyl) trifluoroacetamide plus trimethyl chlorosilane and identified as trimethyl-silyl compounds using a Agilent 7890A GC coupled to a Agilent 5975C MS detector with a capillary column DB-5MS (Agilent, length $30 \mathrm{~m}$, internal diameter $0.25 \mathrm{~mm}$, film $0.25 \mu$ ), with open-split injector and helium as carrier gas. GC/MS temperatures were as follows: column $90^{\circ} \mathrm{C}$ for 5 min until $280^{\circ} \mathrm{C}$ for $10 \mathrm{~min}$ (increment of $3^{\circ} \mathrm{C} / \mathrm{min}$ ), injector $250^{\circ} \mathrm{C}$, transfer line $280^{\circ} \mathrm{C}$, ion source $150^{\circ} \mathrm{C}$, and mass analyzer $35^{\circ} \mathrm{C}$. Finally, mass spectra was programmed from $\mathrm{m} / \mathrm{z} 10$ to 650 at the rate of $0.6 \mathrm{~Hz}$.

\section{Acylcarnitine and amino acid analysis}

Amino acids and acylcarnitines were analyzed in dried blood spot samples by liquid chromatography coupled to mass spectrometry in Tandem (LC/MS/MS) according to Rashed et al. (1997). Blood spot of $3 \mathrm{~mm}$ of diameter were mixed with $100 \mu \mathrm{L}$ of a standard solution of deuterium-labeled acylcarnitines and amino acids. The acylcarnitines and the amino acids were analyzed as butyl esters using a 2695 Alliance (Waters) LC coupled to a Quattro Micro MS/MS (Perkin Elmer). Source and desolvation temperatures were $120^{\circ} \mathrm{C}$ and $300^{\circ} \mathrm{C}$, respectively. Entrance energy, collision energy, and exit energy were $3 \mathrm{~V}, 16 \mathrm{eV}$, and $2 \mathrm{~V}$, respectively. Acylcarnitines and amino acids were monitored by MRM (multiple reaction monitoring), parent ion scan $85(\mathrm{~m} / \mathrm{z})$ or neutral loss scan $102(\mathrm{~m} / \mathrm{z})$. The concentrations were determined by the response of each analyte in relation to their respective labeled internal standard. The results were reported as $\mu \mathrm{mol} / \mathrm{L}$.

\section{Quality control}

Our lab participates in the ERNDIM (European Research Network for Inherited Disorders of Metabolism) schemes for qualitative organic acid, quantitative amino acid, qualitative blood spot acylcarnitine as an external quality control program for accuracy evaluation.

\section{Results}

Samples from $65 \%$ of referred patients came from six states of the southeast and south of Brazil that corresponds to the most prosperous Brazilian regions. We identified 258 cases of organic acid disorders and 117 patients with aminoacidopathies, making a positive diagnostic yield of about $1.72 \%$. Tables 1 and 2 display the sex, average age at diagnosis, and major clinical and laboratory findings of amino acid and organic acid disorders, respectively. The average age at diagnosis was 3.8 years for aminoacidopathies and 4.6 years for organic acidurias. However, most diagnoses of amino acid metabolism disorders occurred before 1 year of age (64\%), whereas $75 \%$ of the children were diagnosed before 3 years. Regarding organic acidurias, $49 \%$ were diagnosed before 1 year of age and $69 \%$ before 3 years. Thus, many patients were diagnosed late with a high proportion of them being misdiagnosed initially as septicemia or meningitis. The delay of diagnosis had resulted in a significant morbidity such as mental retardation and physical handicaps among survivors in this cohort.

Table 3 shows the most prevalent aminoacidopathies and organic acidemias detected in our sample population. It should be emphasized that out of 18 children with urea cycle defects, 15 were diagnosed by LC/MS/MS, whereas only 3 by reverse phase HPLC. Table 4 compares the relative prevalence of organic acidurias in high-risk patients from different populations.

\section{Discussion}

Rare disorders are neglected in most of developing countries due to other common infectious and other more prevalent diseases associated with malnutrition. However, rare disorders need to be investigated because a considerable number of patients in these countries suffers from IEM. Due to the huge burden of IEM, reference centers dedicated to selective screenings are important even for developing nations for the screening and diagnosis of the most prevalent IEMs such as organic acidurias and aminoacidopathies (Sass, 2011). 
Table 1 - Common features of patients with aminoacidopathies detected in high-risk Brazilians.

\begin{tabular}{lc}
\hline Age at diagnosis & Percentage \\
\hline$<1$ month & 12 \\
1 - 12 months & 52 \\
1 - 3 years & 11 \\
3 - 10 years & 14 \\
$>10$ years & 11 \\
\hline Gender & Percentage \\
\hline Female & 45 \\
Male & 55 \\
\hline Clinical features & Percentage \\
\hline Neurological abnormalities & 90 \\
Feeding difficulties & 30 \\
Hepatomegaly & 22 \\
Vomiting & 15 \\
Dysmorphies & 7 \\
Failure to thrive & 3 \\
\hline Neurological signs & Percentage \\
\hline Psychomotor delay/mental retardation & 61 \\
Seizures & 60 \\
Hypotonia/hypertonia/dystonia & 36 \\
Cerebral atrophy & 27 \\
Macrocephaly & 8 \\
\hline Laboratory findings & 41 \\
Metabolic acidosis & 14 \\
Hypoglycemia & \\
Lactic acidemia & 41 \\
\hline
\end{tabular}

Analyses of organic acids and amino acids by sophisticated analytical methods are relatively expensive, especially considering the budget of developing countries. However, because of the relatively high prevalence of organic acidemias and aminoacidopathies among the known IEM (Chalmers et al., 1980) and since many of these diseases can be successfully treated, we decided to implement the techniques for the diagnosis of these diseases at the Medical Genetics Service of the Hospital de Clínicas de Porto Alegre - a regional and national center of reference for studies on IEM. Early recognition of these metabolic diseases is also important when no treatment is currently available, for further family planning and possible prenatal diagnostics. In addition, awareness of these metabolic disorders by pediatricians, neurologists, and other physicians, as well as by the general public is also essential for the development of future therapeutic strategies. Finally, selective screening for IEM enables new disorders to be identified.

Therefore, in the present study, we investigated the relative prevalence of different organic acidurias and
Table 2 - Common features of patients with organic acidurias detected in high-risk Brazilians.

\begin{tabular}{lc}
\hline Age at diagnosis & Percentage \\
\hline$<1$ month & 5 \\
1 - 12 months & 44 \\
1 - 3 years & 20 \\
3 - 10 years & 17 \\
$>10$ years & 14 \\
\hline Gender & Percentage \\
\hline Female & 47 \\
Male & 53 \\
\hline Clinical features & Percentage \\
\hline Neurological & 66 \\
Vomiting & 17 \\
Feeding difficulties & 15 \\
Failure to thrive & 6 \\
Hepatomegaly & 5 \\
Dysmorphies & 4 \\
\hline Neurological signs & Percentage \\
\hline Psychomotor delay/mental retarda- & 30 \\
tion & \\
Seizures & 25 \\
Hypotonia/hypertonia/dystonia & 23 \\
Macrocephaly & 10 \\
\hline Laboratory findings & 32 \\
\hline Metabolic acidosis & 14 \\
Hypoglycemia & \\
& \\
\hline
\end{tabular}

aminoacidopathies in clinically symptomatic Brazilian patients suspected of these pathologies. The major clinical and laboratory findings of the individuals referred to our service included unexplained neurologic signs, feeding difficulties, hepatomegaly, vomiting, failure to thrive, motor disabilities (hypotonia/hypertonia/dystonia), metabolic acidosis, hypoglycemia, and lactic acidosis. We incorporated in our laboratory 15 years ago an international quality control program for organic, amino acid and fatty acid determination that warrants a better reliability of our analysis, implying a good diagnostic approach for these disorders.

The use of a questionnaire containing a large spectrum of clinical and laboratory findings, as well as diet, medication, and age that was filled out by physicians requesting examinations for suspected patients helped us in the diagnosis of these diseases. Thus, these data is utterly important for correct assessment of chromatography findings on amino acids and especially organic acids, considering that there is a high variation on the level of these metabolites pending on age, clinical condition, and treatment at the time of sample collection. In our sample, no clinical and laboratory data were obtained from approximately $20 \%$ of the patients. 
Table 3 - Number and types of aminoacidopathies and organic acidurias detected in high-risk Brazilian patients from 2006 to 2016.

\begin{tabular}{lcc}
\hline Amino acid disorders & Number & Percentage \\
\hline Maple syrup urine disease & 40 & 34 \\
Phenylketonuria & 24 & 21 \\
Non-ketotic hyperglycinemia & 17 & 14 \\
Tyrosinemia & 12 & 10 \\
Homocystinúria & 7 & 6 \\
Citrullinemia & 7 & 6 \\
Ornithine transcarbamylasedeficiency & 4 & 3 \\
Arginase deficiency & 3 & 3 \\
Carbamoyl phosphate synthetase deficiency & 2 & 2 \\
Argininosuccinic aciduria & 1 & 1 \\
Total & 117 & \\
Organic acid disorders & & \\
Lactic acidemia & 64 & 24.8 \\
Glutaric aciduria type I & 59 & 22.8 \\
Methylmalonic aciduria & 40 & 15.5 \\
3-Hydroxy-3-methylglutaric aciduria & 25 & 9.6 \\
Isovaleric aciduria & 12 & 4.6 \\
Propionic aciduria & 12 & 4.6 \\
L-2-hydroxyglutaric aciduria & 11 & 4.2 \\
Alkaptonuria & 10 & 3.8 \\
Canavan disease & 6 & 2.3 \\
3-Methylglutaconic aciduria & 4 & 1.5 \\
D-2-hydroxyglutaric aciduria & 4 & 1.5 \\
3-Ketothiolase deficiency & 3 & 1.5 \\
Mevalonic aciduria & & 1.1 \\
3-Methyl-Crotonyl-CoA Carboxylase defi- & 1.1 \\
ciency & 3.3 \\
Glycerol Kynase deficiency & \\
Total & 3 & \\
\hline & & \\
\hline
\end{tabular}

The average age of diagnosis (when patients were referred to our laboratory) was 3.8 years for aminoacidopathies and 4.6 years for organic acidurias, which could be attributed to lack of awareness of these genetic diseases by the physicians, lack of laboratories and expertise on the diagnosis of these disorders, high patient mortality for some disorders, or a combination of these factors. Nevertheless, the highest proportion of patients was diagnosed in the first year of life, whereas diagnosis of others disorders with lower mortality and less intense clinical manifestations such as L-2-hydroxyglutaric aciduria and glutaric acidemia type I had a late diagnosis.

All cases were confirmed in additional plasma, urine and CSF (patients with nonketotic hyperglycinemia) samples collected on at least two different occasions. For some disorders, we needed additional tests that were carried out in other laboratories especially in Europe and USA. Tan-

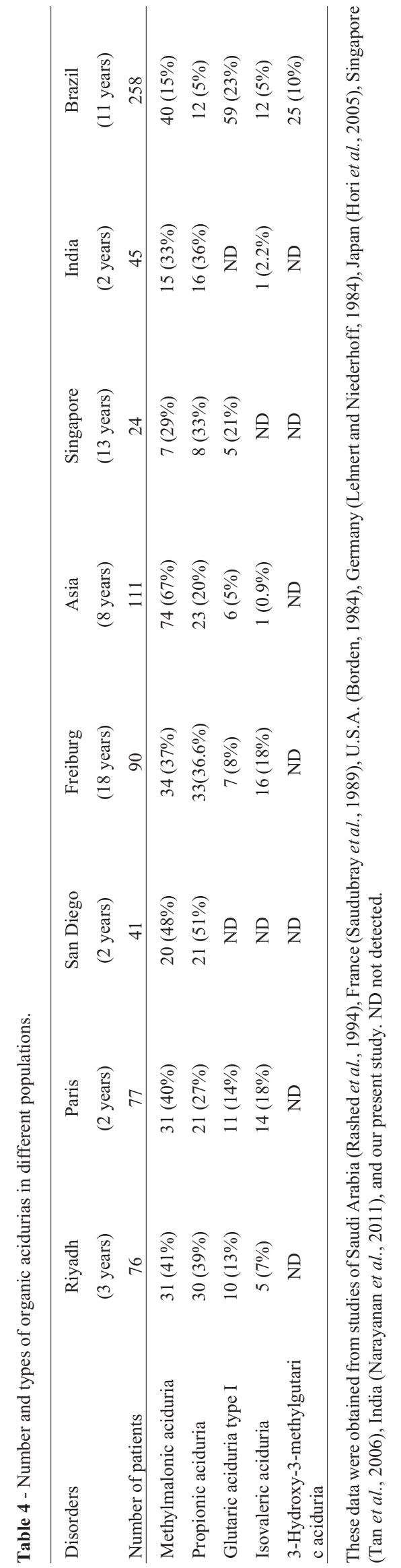


dem MS was also performed in dried blood in some patients suspected of having urea cycle defects since HPLC does not discriminate certain amino acids and this enabled us to diagnose another fifteen cases of aminoacidopathies. Therefore, we strongly recommend this highly analytical method (LC/MS/MS) for the diagnosis of urea cycle defects.

Regarding the relative frequency of organic acidemias in our population, an interesting observation was the high number of glutaric aciduria type I (59 patients) and 3-hydroxy-3-methylglutaric aciduria (40 patients) detected in our study, with a similar prevalence to the other most prevalent organic acidurias, such as methylmalonic acidemia (due to mutase deficiency or various cobalamin deficiencies including $\mathrm{CblC}$ and $\mathrm{Cbl} \mathrm{A}$ ). (Borden, 1984; Lehnert and Niederhoff, 1984; Saudubray et al., 1989; Rashed et al., 1994; Hori et al., 2005; Tan et al., 2006; Narayanan et al., 2011; Al Riyami et al., 2012; Jiang et al., 2015; Lampret et al., 2015). In contrast, the number of propionic acidurias in our population was below the expected and this may have occurred because of the severity and high mortality of the affected patients so that some of them were not referred to our service. Regarding 3-hydroxy-3-methylglutaric aciduria, we have previously reported that some mutations observed in our population were similar to the Portuguese population; by looking at the surnames, all but one patient had Portuguese origin (Vargas et al., 2008).

Of the 64 cases of primary lactic acidemias diagnosed (high lactic aciduria and lactic acidemia) detected on three consecutive occasions, only a few of them had the biochemical and/or the molecular defect defined (including deficiencies of glucose-6-phosphatase deficiency, fructose-1-6-biphosphatase, pyruvate dehydrogenase, and pyruvate carboxylase), indicating the difficulty of establishing the biochemical cause in this group of disorders. Furthermore, approximately half of these children died suddenly at the time of diagnosis or a few weeks later, reflecting the severity of these mitochondrial disorders associated with the lack of effective therapy. On the other hand, since most patients affected by organic acidurias lived in other states in Brazil, we did not have their follow up in order to determine precisely the rate of mortality for these diseases. Nevertheless, all diagnosed patients were immediately submitted to the available treatment mainly based on restricted diets and supplementation of vitamins, amino acid mixture, glycine and/or L-carnitine, and liver transplantation for some those affected by aminoacidopathies as maple syrup urine disease and tyrosinemia type I after all treatment efforts failed. Furthermore, the clinical symptomatology of our patients with organic acidurias was often severe probably because of the late diagnosis of which outcomes are much worse. This was particularly observed in glutaric aciduria type I, in which striatum degeneration affected the vast majority of our patients and represents an irreversible and crucial step towards spasticity and other marked debilitating muscular symptoms.

Another aspect that seems worth mentioning is that specimens for selective organic acid screening need to be collected preferentially during a crisis of metabolic decompensation usually caused by catabolic events triggered by intercurrent infections, changes in nutrition, or prolonged fasting (e.g., the first urine). Sampling after recovery, even if it is only partial, may result in decreased sensitivity and may not reveal diagnostic abnormalities detectable before recovery.

Regarding aminoacidopathies, we diagnosed a relatively high number of maple syrup urine disease (40), nonketotic hyperglycinemia (17), and tyrosinemia type I (12), probably because of the severity of the clinical findings of these disorders, which raises the awareness of pediatricians and neurologists. Of the urea cycle defects cases, only three were diagnosed by HPLC, and 15 additional patients with these disorders were diagnosed by LC/MS/MS. Thus, it is emphasized that although important, HPLC does not allow complete separation and/or identification of all amino acids that can be otherwise identified by amino acid analysis on LC/MS/MS, including arginine and citruline. Interestingly, 20 cases of PKU were referred to our laboratory for confirmation because of abnormal tests (high phenylalanine levels) in the first sample at private laboratories also dedicated to the NBS program, whereas we diagnosed four cases of PKU in mentally retarded children that probably escaped the NBS detection of this disorder, whose nationwide coverage is about $86 \%$ in Brazilians babies.

Finally, we emphasize that some patients with organic acid disorders and aminoacidopathies were inadvertently confounded with septicemia, which also presents severe symptoms such as acidosis and acute encephalopathy; these late diagnoses often lead to death or permanent sequelae. Our results indicate the importance of diagnosing organic acidemias and aminoacidopathies in loco and as early as possible even in a developing country and particularly in severely ill patients because of the high prevalence of these diseases in these high-risk groups of patients, and particularly because of the availability of therapy for many of them. Treatment monitoring of affected individuals by serial analysis of urine and blood metabolites is also important and justifies the setup of such facilities despite extra costs. Our study shows that amino acids and organic acids disorders may warrant the development of a nationwide screening program for the diagnosis of these disorders because of their high incidence in the neonatal period in Brazil. Thus, we propose that the NBS program should be expanded in Brazil to include these disorders, besides PKU, in neonates in order to avoid the high morbidity and mortality, ensuring easy and equal access to this preventive public health initiative and to increase the awareness and knowledge among the general public and especially, health professionals. Further studies are needed to investigate the 
long-term outcome and costs associated with the diagnosis and treatment of these disorders.

\section{Acknowledgments}

We gratefully acknowledge the financial support of FIPE/HCPA, Conselho Nacional de Desenvolvimento Científico e Tecnológico (CNPq) (Brasília, Brazil), Financiadora de Projetos (FINEP) (Brasília, Brazil), Fundação de Amparo à Pesquisa do Rio Grande do Sul (FAPERGS) (Porto Alegre, Brazil), and Pró-Reitoria de Pesquisa/ UFRGS (PROPESP/UFRGS) (Porto Alegre, Brazil). We would like also to thank all staff, but especially the doctors of the Medical Genetics Service of the Clinical Hospital of Porto Alegre, as well as the physicians from the other hospitals of Brazil. Finally, we thank Marcelo Soares for his excellent technical assistance.

\section{Conflict of Interest}

The authors declare that there is no conflict of interest related to this study.

\section{Author contributions}

MW conceived the study and contributed with all sections; Angela Sitta performed the amino acid, organic acid and acylcarnitine analyses and contributed with Results and statistical calculations; AK and ACG performed the amino acid and organic acid analyses and with the statistical calculations; MD performed the acylcarnitine analyses and contributed with Results and statistical calculations; DMC performed the amino acid and organic acid and contributed with statistical calculations and results; CRV contributed with all sections.

\section{References}

Al Riyami S, Al Maney M, Joshi SN and Bayoumi R (2012) Detection of inborn errors of metabolism using tandem mass spectrometry among high-risk Omani patients. Oman Med J 27:482-485.

Borden M (1984) Screening for metabolic disorders. In: Nyhan WI (ed) Amino Acid Metabolism in Clinical Medicine. Appleton-Century-Crofts, Norwalk, pp 401-417.

Bravo H, Neto EC, Schulte J, Pereira J, Filho CS, Bittencourt F, Sebastião F, Bender F, de Magalhães APS, Guidobono R et al. (2017) Investigation of newborns with abnormal results in a newborn screening program for four lysosomal storage diseases in Brazil. Mol Genet Metab Rep 12:92-97.

Chalmers RA, Purkiss P, Watts RW and Lawson AM (1980) Screening for organic acidurias and amino acidopathies in newborns and children. J Inherit Metab Dis 3:27-43.

García-Gazorla A, Wolf NI, Serrano M, Moog U, Pérez-Dueñas B, Póo P, Pineda M, Campistol J and Hoffmann GF (2009) Mental retardation and inborn errors of metabolism. J Inherid Metab Dis 32:597-608.

Gramer G, Haege G, Glahn EM, Horffmann GF, Lindner M and Burgard P (2014) Living with an inborn error of metabolism detected by newborn screening-parents' perspectives on child development and impact on family life. J Inherit Metab Dis 37:189-195.

Hori D, Hasegawa Y, Kimura M, Yang Y, Verma IC and Yamaguchi S (2005) Clinical onset and prognosis of Asian children with organic acidemias, as detected by analysis of urinary organic acids using GC/MS, instead of mass screening. Brain Dev 27:39-45.

Jiang M, Liu L, Mei H, Li X, Cheng J and Cai Y (2015) Detection of inborn errors of metabolism using GC-MS: Over 3 years of experience in southern China. J Pediatr Endocrinol Metab 28:375-380

Joseph MH and Marsden CA (1986) Amino acids and sample peptides. In: Lim CK (ed) HPLC of Small Peptides. IRL Press, Oxford, pp 13-27.

Lampret BR, Murko S, Tanek M, Podkrajek KT, Debeljak M, mon A and Battelino T (2015) Selective screening for metabolic disorders in the Slovenian pediatric population. Med Biochem 34:58-63.

Lehnert W and Niederhoff H (1984) Seven years of experience with selective screening for organic acidurias. Eur J Pediatr 142:208-210.

Leonard JV (2006) Komrower lecture: Treatment of inborn errors of metabolism: A review. J Inherit Metab Dis 29:275-278.

Mak CM, Lee HC, Chan AY and Lam CW (2013) Inborn errors of metabolism and expanded newborn screening: Review and update. Crit Rev Clin Lab Sci 50:142-162.

Marsdeen D, Lason DC and Levy HL (2006) Newborn screening for metabolic disorders. J. Pediatr 148:577-584.

Narayanan MP, Kannan V, Vinayan KP and Vasudevan DM (2011) Diagnosis of major organic acidurias in children: Two years experience at a tertiary care centre. Indian J Clin Biochem 26:347-353.

Rashed M, Ozand PT, Aqeel A and Gascon GG (1994) Experience of King Faisal specialists hospital and research center with Saudi organic acid disorders. Brain Dev 16 Suppl:1-6.

Rashed M, Bucknall M, Little D, Awad A, Jacob M, Alamoudi M, Almattar M and Ozand PT (1997) Screening blood spots for inborn errors of metabolism by electrospray tandem mass spectrometry with a microplate batch process and a computer algorithm for automated flagging of abnormal profiles. Clin Chem 43:1127-1141.

Sass JO (2011) Selective screening for inborn errors of metabolism-assessment of metabolites in body fluids. Clin Biochem 44:474-475.

Saudubray JM, Ogier H, Bonnefont JP, Munnich A, Lombes A, Herve F, Mitchel G, Thé BP, Specola N, Parvy P et al. (1994) Clinical approach to inherited metabolic diseases in the neonatal period: A 20 years survey. J Inher Metab Dis 12 Suppl 1:25-41.

Scaturro G, Sanfilippo C, Picciome M, Piro E, Giuffre M and Consello G (2013) Newborn screening of inherited metabolic disorders by tandem mass spectrometry: Past, present and future. Pediatr Med Chir 35:105-109.

Scriver CR, Beaudet A, Sly WS and Valle D (2001) The Metabolic and Molecular Bases of Inherited Disease. 8th edition. McGraw-Hill, New York, 6338 p.

Sweetmann L (2006) Organic acid analysis. In: Hommes FA (ed) Techniques in Diagnostic Human Biochemical Genetics: A laboratory manual. Wiley-Liss, New York, pp 143-176. 
Tan IK, Gajra B and Lim MS (2006) Study of inherited metabolic disorders in Singapore - 13 years experience. Ann Acad Med Singapore 35:804-813.

Van Vliet D, Derks TG, Van Rijn M, de Groot MJ, MacDonald A, Heiner-Fokkema MR and Van Spronsen FJ (2014) Single amino acid supplementation in aminoacidopathies: A systematic review. Orphanet J Rare Dis 9:7.

Vargas CR, Sitta A,Schmitt G,Ferreira GC,Cardoso ML,Coelho D,Gibson KM andWajner M (2008) Incidence of 3-hydroxy-3-methylglutaryl-coenzyme A lyase (HL) deficiency in Brazil, South America. J Inherit Metab DisSuppl 3:511-515.

Wajner M, Coelho DM, Barschak AG, Araujo PR, Pires RF, Lulhier FL and Vargas CR (2000) Reduction of large neutral amino acid concentrations in plasma and CSF of patients with maple syrup urine disease during crises. J Inherit Metab Dis 23:505-512.

Associate Editor: Roberto Giugliani

License information: This is an open-access article distributed under the terms of the Creative Commons Attribution License (type CC-BY), which permits unrestricted use, distribution and reproduction in any medium, provided the original article is properly cited. 